\title{
A note on the good lambda inequalities ${ }^{\dagger}$
}

\author{
S.D. Jacka \\ Department of Statistics \\ University of Warwick \\ Coventry \\ CV4 7AL \\ UK
}

\section{$\S 1$. Introduction}

1.1 The celebrated good-lambda inequalities of the form

$$
\mathbb{P}\left(X_{T} \geq \beta \lambda ; Y_{T}<\delta \lambda\right) \leq c(\beta, \delta) \mathbb{P}\left(X_{T} \geq(\beta-1) \lambda\right)
$$

and which are due to Burkholder (see Burkholder (1973)), play a crucial role in deducing inequalities of the form

$$
\left\|X_{T}\right\|_{p} \leq C_{p}\left\|Y_{T}\right\|_{p} \quad \forall \text { optional } T,
$$

or, more generally,

$$
\mathbb{E} F\left(X_{T}\right) \leq C_{F} \mathbb{E} F\left(Y_{T}\right) \quad \forall \text { optional } T
$$

where $X$ and $Y$ are positive increasing previsible processes and $F$ is a moderate function (see, for example, Azéma, Gundy and Yor(1980), Bass (1987), Davis (1987), Barlow and Yor (1982), and the seminal paper by Lenglart, Lépingle and Pratelli (1980)).

Inequalities such as (1.1.1) are deduced by proving that the constant $c(x, y ; z)$, appearing in

$$
\mathbb{P}\left(X_{T} \geq x ; Y_{T}<y\right) \leq c(x, y ; z) \mathbb{P}\left(X_{T} \geq z\right) \quad \forall \text { optional } T
$$

has a suitable form. The main result of this paper is

Theorem 5. If $X$ is right-continuous and previsible then if $x>z>X_{0}$, the best constant appearing in (1.1.2) is

$$
c(x, y ; z)=\left\|\mathbb{P}\left(X_{\infty} \geq x ; Y_{S_{x}}<y \mid \mathcal{F}_{S_{z}-}\right)\right\|_{\infty}
$$

where $S_{u}=\inf \left\{t \geq 0: X_{t} \geq u\right\}$.

1.2 Many interesting processes in martingale theory satisfy the conditions of Theorem 5 and are time-changes of a sub-additive functional of an underlying Brownian motion.

$\dagger$ This paper appeared in Séminaire de Probabilités XXIII 57-65, LNM 1372, Springer (1989). 
As we shall see in section 3 , finding an upper bound for $c(x, y ; z)$ then reduces to finding $p(x-z, y)$ where $p(\cdot, \cdot)$ is given by:

$$
\begin{aligned}
p(x, y) & =\sup _{\text {optional } T} \mathbb{P}\left(X_{T} \geq x ; Y_{T}<y\right) \\
& =\mathbb{P}\left(X_{\infty} \geq x ; Y_{S_{x}}<y\right)
\end{aligned}
$$

Thus verifying the good lambda inequalities reduces to finding good bounds for $p(x, y)$. Indeed, we shall see in section 4 that better results may be achieved by this direct approach than have been derived to date.

\section{$\S 2$. Good lambda inequalities}

We assume throughout this section that $X$ is an increasing process adapted to the filtered probability space $\left(\Omega, \mathcal{F},\left(\mathcal{F}_{t} ; t \geq 0\right), \mathbb{P}\right)$ satisfying the usual conditions and $Y$ is an increasing $\mathcal{F}$-measurable process.

We define $\mathcal{T}=\{$ optional (stopping) times $T\}$.

2.1 We consider first an upper bound for $c(x, y, z)$.

Define $S_{u}=\inf \left\{t \geq 0: X_{t} \geq u\right\}$.

Lemma 2 Suppose $X$ is a.s. right-continuous and $x>z$ then for any $T \in \mathcal{T}$ :

$$
\mathbb{P}\left(X_{T} \geq x ; Y_{T}<y\right) \leq\left\|\mathbb{P}\left(X_{S_{x}} \geq x ; Y_{S_{x}}<y \mid \mathcal{F}_{S_{z}-}\right)\right\|_{\infty} \mathbb{P}\left(X_{T} \geq z\right)
$$

so that $c(x, y ; z) \leq\left\|\mathbb{P}\left(X_{S_{x}} \geq x ; Y_{S_{x}}<y \mid \mathcal{F}_{S_{z}-}\right)\right\|_{\infty}$.

Proof: $\quad$ Fix $T$ and set $p=\mathbb{P}\left(X_{T} \geq x ; Y_{T}<y\right)$

Since $X$ is right-continuous $\left(X_{T} \geq x\right)=\left(T \geq S_{x}\right) \cap\left(X_{S_{x}} \geq x\right)$, and since $Y$ is increasing

$$
\left(Y_{T}<y\right) \subset\left(Y_{S_{x}}<y\right) \text { on }\left(T \geq S_{x}\right)
$$

so

$$
p \leq \mathbb{P}\left(X_{S_{x}} \geq x ; Y_{S_{x}}<y ; T \geq S_{x}\right)
$$

Moreover $\left(X_{S_{x}} \geq x\right) \subset\left(X_{S_{z}} \geq z\right)$ (since $\left.z<x\right)$ and so, defining

$$
\begin{gathered}
Z=\mathbb{P}\left(X_{S_{x}} \geq x ; Y_{S_{x}}<y \mid \mathcal{F}_{S_{z}-}\right) \\
p \leq \mathbb{E}\left[\mathbb{E}\left[1_{\left(X_{S_{x}} \geq x\right) \cap\left(Y_{S_{x}}<y\right)} 1_{\left(T \geq S_{z}\right) \cap\left(X_{S_{z}} \geq z\right)} \mid \mathcal{F}_{S_{z}}\right]\right]
\end{gathered}
$$

Now, since $T$ is optional, $\left(T \geq S_{z}\right) \in \mathcal{F}_{S_{z}-}$ (see Dellacherie and Meyer (1978) Theorem 56 ), whilst $\left(X_{S_{z}} \geq z\right)=\left(S_{z}<\infty\right) \cup\left(\left(S_{z}=\infty\right) \cap\left(\lim _{t \rightarrow \infty} X_{t} \geq z\right)\right) \in \mathcal{F}_{S_{z}-}$ (by Theorem 56(e) of Dellacherie and Meyer), so

$$
\begin{aligned}
p & \leq \mathbb{E} Z 1_{\left(X_{T} \geq z\right)} \\
& \leq\|Z\|_{\infty} \mathbb{P}\left(X_{T} \geq z\right)
\end{aligned}
$$


2.2 Since this author is happiest dealing with continuous processes we shall first look at the converse of Lemma 2 when $X$ is continuous.

First, we define

$$
S_{u}^{\prime}=\inf \left\{t \geq 0: X_{t}>u\right\}
$$

Lemma 3 Suppose $X$ is continuous, $x>w>X_{0}$, and $\mathbb{P}\left(X_{\infty}>w\right)>0$ then

$$
r \equiv \frac{\sup _{T \in \mathcal{T}} \mathbb{P}\left(X_{T} \geq x ; Y_{T}<y\right)}{\mathbb{P}\left(X_{T}>w\right)} \geq\left\|\mathbb{P}\left(X_{S_{x}} \geq x ; Y_{S_{x}}<y \mid \mathcal{F}_{S_{w}^{\prime}}\right)\right\|_{\infty} .
$$

Proof: Define

$$
Z_{w}=\mathbb{P}\left(X_{S_{x}} \geq x: Y_{S_{x}}<y \mid \mathcal{F}_{S_{w}^{\prime}}\right)
$$

and assume wlog that

$$
d \equiv\left\|\mathbb{P}\left(X_{S_{x}} \geq x ; Y_{S_{x}}<y \mid \mathcal{F}_{S_{w}^{\prime}}\right)\right\|_{\infty}>0 .
$$

Define, for each $a \in(0, d)$ :

$$
\tau_{a}=S_{w}^{\prime} 1_{\left(Z_{w}<a\right)}+S_{x} 1_{\left(Z_{w} \geq a\right)}
$$

Now, by continuity $X_{S_{w}^{\prime}} \leq w$ so

$$
\mathbb{P}\left(X_{\tau_{a}}>w\right)=\mathbb{P}\left(Z_{w} \geq a ; X_{S_{x}}>w\right)
$$

Now, since $a>0$, we see that $\left(Z_{w} \geq a\right) \subset\left(S_{w}^{\prime}<\infty\right) \subset\left(X_{S_{x}}>w\right)$ so

$$
\mathbb{P}\left(X_{\tau_{a}}>w\right)=\mathbb{P}\left(Z_{w} \geq a\right)
$$

moreover

$$
\begin{aligned}
& \mathbb{P}\left(X_{\tau_{a}} \geq x ; Y_{\tau_{a}}<y\right) \\
= & \mathbb{P}\left(X_{S_{x}} \geq x ; Y_{S_{x}}<y ; Z_{w} \geq a\right) \\
= & \mathbb{E}\left\{\mathbb{E}\left(1_{\left(X_{S_{x}} \geq x\right) \cap\left(Y_{S_{x}}<y\right)} 1_{\left(Z_{w} \geq a\right)} \mid \mathcal{F}_{S_{w}^{\prime}}\right)\right\} \\
= & \mathbb{E} Z_{w} 1_{\left(Z_{w} \geq a\right)}
\end{aligned}
$$

so that $r \geq \mathbb{E} Z_{w} 1_{\left(Z_{w} \geq a\right)} / \mathbb{P}\left(Z_{w} \geq a\right)$ and letting $a \uparrow \uparrow\left\|Z_{w}\right\|_{\infty}$ we obtain the result.

We may obtain the following corollary by letting $w$ increase (strictly) to $z$ and by observing that $S_{z}=\lim _{w \uparrow \uparrow z} S_{w}^{\prime}$

Corollary 4 If $X$ is continuous and $x>z>X_{0}$ then

$$
c(x, y ; z)=\left\|\mathbb{P}\left(X_{S_{x}} \geq x ; Y_{S_{x}}<y \mid \mathcal{F}_{S_{z}-}\right)\right\|_{\infty} .
$$

Proof: From lemma 2 we need only prove that

$$
c(x, y ; z) \geq\left\|\mathbb{P}\left(X_{S_{x}} \geq x ; Y_{S_{x}}<y \mid \mathcal{F}_{S_{z}-}\right)\right\|_{\infty} .
$$


Now

$$
\begin{gathered}
c(x, y ; z)=\sup _{T \in \mathcal{T}} \frac{\mathbb{P}\left(X_{T} \geq x ; Y_{T}<y\right)}{\mathbb{P}\left(X_{T} \geq z\right)} \\
\left.=\sup _{T \in \mathcal{T}} \sup _{w<z} \frac{\mathbb{P}\left(X_{T} \geq x ; Y_{T}<y\right)}{\mathbb{P}\left(X_{T}>w\right)} \quad \text { (since } \mathbb{P}\left(X_{T}>w\right) \downarrow \mathbb{P}\left(X_{T} \geq z\right) \text { as } w \uparrow \uparrow z\right) \\
=\sup _{w<z} \sup _{T \in \mathcal{T}} \frac{\mathbb{P}\left(X_{T} \geq x ; Y_{T}<y\right)}{\mathbb{P}\left(X_{T}>w\right)} \\
\geq \sup _{w<z}
\end{gathered}
$$

Now $\left(Z_{w} ; w<z\right)$ is a bounded martingale on the filtration $\left(\mathcal{G}_{w} ; w<z\right)=\left(\mathcal{F}_{S_{w}^{\prime}} ; w<z\right)$ and so, by the bounded martingale convergence theorem, $Z_{w} \stackrel{a . s .}{\longrightarrow} Z$ where

and

$$
Z=\mathbb{P}\left(\left(X_{S_{x}} \geq x ; Y_{S_{x}} \leq y\right) \mid \mathcal{G}\right)
$$

$$
\mathcal{G}=\bigvee_{w<z} \mathcal{G}_{w}=\bigvee_{w<z} \mathcal{F}_{S_{w}^{\prime}}
$$

Now $S_{w}^{\prime} \uparrow \uparrow S_{z}$ as $w \uparrow \uparrow z$ so, by theorem 56 of Dellacherie and Meyer (1978), $\mathcal{G}=\mathcal{F}_{S_{z}-}$ and so $Z$ is as defined in Lemma 2 . Thus since $\left\|Z_{w}\right\|_{\infty}$ is increasing, $\sup _{w<z}\left\|Z_{w}\right\|_{\infty}=$ $\|Z\|_{\infty}$ establishing (2.2.1).

2.3 We can, with a little more effort, deal with the case where $X$ is previsible and right-continuous. The trick is to observe that if $X$ satisfies these conditions then $S_{z}$ is previsible so we can mimic the $\left(S_{w}\right)$ optional times by a sequence $\left(T_{n}\right)$ which announces $S_{z}$.

Theorem 5 Suppose that $X$ is previsible and right-continuous, then if $x>z>X_{0}$

$$
c(x, y ; z)=\left\|\mathbb{P}\left(X_{S_{x}} \geq x ; Y_{S_{x}}<y \mid \mathcal{F}_{S_{z}}\right)\right\|_{\infty} .
$$

Using the same definitions for $Z$ and $d$ as in the proof of lemma 3 we need only prove that (2.2.1) still holds. We need first to establish the following lemma.

Lemma 6 Under the conditions of Theorem 5 the optional time $S_{z}$ is previsible.

Proof We need to prove that the stochastic interval $\llbracket S_{z}, \infty \llbracket \in \mathcal{P}$, the previsible $\sigma$-field (see Dellacherie and Meyer (1978)). Now, since $X$ is right-continuous,

$$
A=\llbracket S_{z}, \infty \llbracket=\left\{(t, w): X_{t}(w) \geq z\right\}
$$

and so, since $X$ is previsible, $A \in \mathcal{P}$.

Proof of Theorem 5: Since $Z>X_{0}$ and $X$ is right-continuous $S_{z}>0$, and so we can take a sequence of previsible times $T_{n}$ such that $T_{n} \uparrow \uparrow S_{z}($ a.s.) as $n \rightarrow \infty$ 
(Dellacherie and Meyer (1978), Theorem 71). Note that, since $T_{n}<S_{z}$ a.s. and $X$ is right-continuous, $X_{T_{n}}<Z$ a.s. Fix $n$ and $a \in(0, d)$ (as before $d>0$ wlog) and define

$$
Z_{n}=\mathbb{P}\left(X_{S_{x}} \geq x ; Y_{S_{x}}<y \mid \mathcal{F}_{T_{n}}\right)
$$

and

$$
\tau=T_{n} 1_{\left(Z_{n}<a\right)}+S_{x} 1_{\left(Z_{n} \geq a\right)} .
$$

We see that

$$
\begin{gathered}
\frac{\mathbb{P}\left(X_{\tau} \geq x ; Y_{\tau}<y\right)}{\mathbb{P}\left(X_{\tau} \geq z\right)} \\
=\mathbb{E}\left(1_{\left(X_{S_{x}} \geq x ; Y_{S_{x}}<y\right)} 1_{\left(Z_{n} \geq a\right)}\right) \mid \mathbb{P}\left(Z_{n} \geq a ; X_{S_{x}} \geq z\right) \\
\geq \frac{\mathbb{E} Z_{n} 1_{\left(Z_{n} \geq a\right)}}{\mathbb{P}\left(Z_{n} \geq a\right)}=r(n, a)
\end{gathered}
$$

Letting $a \uparrow \uparrow d_{n} \equiv\left\|Z_{n}\right\|_{\infty}$ we see that $c(x, y ; z) \geq\left\|Z_{n}\right\|_{\infty}$, and, letting $n \rightarrow \infty$ we obtain (2.2.1) using the same argument as in corollary 4 since $T_{n} \uparrow \uparrow S_{z}$ a.s. and so $\bigvee_{n} \mathcal{F}_{T_{n}}=\mathcal{F}_{S_{z}-}$

Remark: note that we could still conclude, if $X$ was just right-continuous and not previsible that

$$
c(x, y ; z) \geq\left\|\mathbb{P}\left(X_{S_{x}} \geq x ; Y_{S_{x}}<y \mid \mathcal{F}_{T}\right)\right\|
$$

for any optional time $T<S_{z}$, the predictability of $X$ was only used to ensure the existence of $T_{n} \uparrow \uparrow S_{z}$ a.s.

2.4 One might ask whether any lower bound for $c(x, y, z)$ can be given when $X$ is not previsible: if we're prepared to work with $\left(X_{t-}\right)$ we can get somewhere. Note that, setting $X_{t}^{\prime}=X_{t-}, X^{\prime}$ is previsible since it is left continuous.

Theorem 7 Define

$$
c^{\prime}(x, y ; z)=\sup _{T \in \mathcal{T}} \frac{\mathbb{P}\left(X_{T-} \geq x ; Y_{T}<y\right)}{\mathbb{P}\left(X_{T-}>z\right)}
$$

then, if $x>z>X_{0}$,

$$
c^{\prime}(x, y ; z) \geq\left\|\mathbb{P}\left(X_{S_{x}+} \geq x ; Y_{S_{x}+}<y \mid \mathcal{F}_{S_{z}^{\prime}}\right)\right\|_{\infty}
$$

where $S_{z}^{\prime}$ is, as before, $\inf \left\{t \geq 0: X_{t}>z\right\}$.

Proof: Note that, by left-continuity,

$$
X_{S_{z}^{\prime}}^{\prime} \equiv X_{S_{z}^{\prime}-} \leq z
$$

Define $Z=\mathbb{P}\left(X_{S_{x}+} \geq x ; Y_{S_{x}+}<y \mid \mathcal{F}_{S_{z}^{\prime}}\right)$. We assume wlog that

$$
0<d=\|Z\|_{\infty}
$$


and, fixing $a \in(0, d), \varepsilon>0$; we define

$$
\tau \equiv \tau_{\varepsilon}^{a}=S_{z}^{\prime} 1_{(Z<a)}+\left(S_{x}+\varepsilon\right) 1_{(Z \geq a)} 1_{\left(S_{x}<\infty\right)}+S_{x} 1_{(Z \geq a)} 1_{\left(S_{x}=\infty\right)} .
$$

Now $\mathbb{P}\left(X_{\tau-}>z\right)=\mathbb{P}\left(Z \geq a ; X_{\tau-}>z\right) \leq \mathbb{P}(Z \geq a)$, whilst

$$
\begin{aligned}
& \mathbb{P}\left(X_{\tau-} \geq x, Y_{\tau}<y\right) \\
& \quad= \\
& \mathbb{P}\left(Z \geq a ;\left(X_{\left(S_{x}+\varepsilon\right)-} \geq x ; Y_{S_{x}+\varepsilon}<y, S_{x}<\infty\right) \cup\left(X_{\infty-} \geq x, Y_{\infty}<y, S_{x}=\infty\right)\right):
\end{aligned}
$$

if we now let $\varepsilon \downarrow \downarrow 0$ (observing that $X_{\infty_{-}}=X_{\infty} \stackrel{\text { def }}{=} X_{\infty_{+}}$), we see that

$$
c^{\prime}(x, y ; z) \geq \frac{\mathbb{P}\left(Z \geq a ; X_{S_{x}+} \geq x ; Y_{S_{x}+}<y\right)}{\mathbb{P}(Z \geq a)}
$$

and letting $a \uparrow \uparrow d$ we obtain the result.

We can obtain a new converse to (2.4.1) as follows.

Theorem 8 Suppose $X$ is right-continuous and $x>X_{0}$ then

$$
c^{\prime}(x, y ; z) \leq\left\|\mathbb{P}\left(X_{S_{x}} \geq x ; Y_{S_{x}-}<y \mid \mathcal{F}_{S_{z}^{\prime}}\right)\right\|_{\infty} .
$$

Proof: take $z<w<x$ and define

$$
Z_{w}=\mathbb{P}\left(X_{S_{w}^{\prime}} \geq w ; Y_{S_{w}^{\prime}+}<y \mid \mathcal{F}_{S_{z}^{\prime}}\right)
$$

Now for any $\tau \in \mathcal{T}: \mathbb{P}\left(X_{\tau-}>w ; Y_{\tau}<y\right)$

$$
\begin{aligned}
& =\mathbb{P}\left(\tau>S_{w}^{\prime} ; Y_{\tau}<y\right) \\
& \leq \mathbb{P}\left(\tau>S_{w}^{\prime} ; Y_{S_{w}^{\prime}}<y\right) \\
& \leq \mathbb{P}\left(X_{S_{w}^{\prime}} \leq w ; Y_{S_{w}^{\prime}}<y ; \tau>S_{z}^{\prime}\right) \\
& \leq\left\|Z_{w}\right\|_{\infty} \mathbb{P}\left(\tau>S_{z}^{\prime}\right)\left(\text { since }\left(\tau>S_{z}^{\prime}\right) \in \mathcal{F}_{S_{z}^{\prime}}\right) \\
& =\left\|Z_{w}\right\|_{\infty} \mathbb{P}\left(X_{\tau-}>z\right) .
\end{aligned}
$$

Now $S_{w}^{\prime} \uparrow S_{x}$ (not necessarily strictly) whilst $\left(X_{S_{w}^{\prime}} \geq w\right)=\left(X_{\infty} \geq w\right)$ so $\left(X_{S_{x}} \geq x\right) \equiv$ $\left(X_{\infty} \geq x\right)=\bigcap_{z<w<x}\left(X_{\infty} \geq w\right)=\lim _{w \uparrow \uparrow x}\left(X_{\infty} \geq w\right)=\lim _{w \uparrow \uparrow x}\left(X_{S_{w}^{\prime}} \geq w\right)$, thus

$$
\left(X_{S_{x}} \geq x ; Y_{S_{x}-}<y\right) \supset \lim _{w \uparrow \uparrow x}\left(X_{S_{w}^{\prime}} \geq w, Y_{S_{w}^{\prime}}<y\right)
$$

and so we obtain the result. 


\section{$\S 3$. Application to sub-additive functionals}

3.1 We suppose now that $X$ and $Y$ are non-negative increasing functionals of an underlying Markov process $\left(\xi_{t}(\omega), \theta_{t} ; t \geq 0 ; \mathbb{P}_{\eta}, \eta \in E\right)$, where $\left(\theta_{t}\right)$ is the family of shift operators, and $X$ is continuous. Furthermore we suppose that

$$
X_{t}(\omega)-X_{s}(\omega) \leq K_{1} X_{t-s} \circ \theta_{s}(\omega) \forall s, t,
$$

(note that, since $X \geq 0$ and increasing, (3.1.1) is trivially satisfied for $t \leq s$ ), and

$$
Y_{t-s} \circ \theta_{s} \leq K_{2} Y_{t}
$$

(see Bass (1987) for an application of these conditions).

Under these additional conditions we have the following theorem.

Theorem 9 The constant $c(x, y ; z)$ appearing in Lemma 2 has the following upper bound:

$$
c(x, y ; z) \leq p^{*}\left(\frac{x-z}{K_{1}}, K_{2} y\right)
$$

where

$$
p^{*}(u, v)=\sup _{\eta \in E} \mathbb{P}_{\eta}\left(X_{S_{u}} \geq u, Y_{S_{u}} \leq v\right)
$$

Proof: Define $T_{y}=\inf \left\{t \geq 0: Y_{t} \geq y\right\}$ and $\delta=(x-z) / K_{1}$. From lemma 2 we need only bound $\left\|\mathbb{P}\left(X_{S_{x}} \geq x, Y_{S_{x}}<y \mid \mathcal{F}_{S_{z}-}\right)\right\|_{\infty}$ which is dominated by $\| \mathbb{P}\left(X_{S_{x}} \geq x ; Y_{S_{x}}<\right.$ $\left.y \mid \mathcal{F}_{S_{z}}\right) \|_{\infty}$. Now

$$
\begin{array}{rlr} 
& \mathbb{P}\left(X_{S_{x}} \geq x ; Y_{S_{x}}<y \mid \mathcal{F}_{S_{z}}\right) & \\
\leq & \mathbb{P}\left(X_{T_{y}} \geq x \mid \mathcal{F}_{S_{z}}\right) & \\
\leq & \mathbb{P}\left(X_{T_{y}}-X_{S_{z}} \geq x-z \mid \mathcal{F}_{S_{z}}\right) & \text { (by continuity) } \\
\leq & \mathbb{P}\left(X_{\left(T_{y}-S_{z}\right)(\omega)} \circ \theta_{S_{z}(\omega)} \geq \frac{x-z}{K_{1}} \mid \mathcal{F}_{S_{z}}\right) & \text { (by (3.1.1)) } \\
\leq \mathbb{P}\left(X_{T_{K_{2}}\left(\theta_{S_{z}}(\omega)\right)} \circ \theta_{S_{z}(\omega)} \geq s \mid \mathcal{F}_{S_{z}}\right) & \text { (by (3.1.2)) } \\
= & \mathbb{P}_{\xi_{S_{z}}\left(X_{T_{K_{2}} y} \geq \delta\right)} \\
\leq & \sup _{\eta \in E} \mathbb{P}_{\eta}\left(X_{S_{\delta}} \geq \delta ; Y_{S_{\delta}} \leq K_{2} y\right), &
\end{array}
$$

and,taking the essential supremum of the left hand side of this inequality we obtain the result 


\section{$\S 4$. General applications}

4.1 We need the normal form of the good lambda inequalities.

Theorem 10 Suppose $X$ and $Y$ are non-negative r.v.s satisfying:

$$
\mathbb{P}(X \geq \beta \lambda ; Y \leq \delta \lambda) \leq c(\beta, \delta) \mathbb{P}(X \geq \lambda) \quad \forall \lambda>0
$$

then

(i) for any increasing function $F$ with $F(0)=0$, for any $\beta>1, \delta>0$,

$$
\mathbb{E} F(X \wedge N) \leq \mathbb{E} F\left(\frac{\beta Y}{\delta} \wedge N\right)+c(\beta, \delta) \mathbb{E} F(\beta X \wedge N),
$$

(ii) if $F$ is moderate with exponent $p$ (i.e. $\sup _{x>0} \sup _{\alpha>1} \frac{F(\alpha x)}{\alpha^{p} F(x)} \leq 1$ ) then, if $\exists \hat{\delta} \geq 0, \hat{\beta}>1$ s.t. $\hat{\beta}^{p} c(\hat{\beta}, \hat{\delta})<1$,

$$
\mathbb{E} F(X) \leq\left(1 \vee\left(\frac{\hat{\beta}}{\hat{\delta}}\right)^{p}\right) \mathbb{E} F(Y) /\left(1-\hat{\beta}^{p} c(\hat{\beta}, \hat{\delta})\right) .
$$

See Lenglart, Lépingle and Pratelli (1980) for the proof.

4.2 Many pairs of increasing functionals of Brownian motion satisfy

$$
p^{*}(x, y) \leq k \exp (-\theta x / y)
$$

where $p^{*}$ is given by (3.1.4)

Theorem 11 Suppose $X$ and $Y$ satisfy the conditions of Theorem 9 and (4.2.1) then

$$
\begin{gathered}
\left\|X_{T}\right\|_{p} \leq C\left(p, k ; \frac{\theta}{K_{1} K_{2}}\right)\left\|Y_{T}\right\|_{p} \quad \forall \text { optional } T \\
\text { and } C_{p} \equiv C\left(p, k ; \frac{\theta}{K_{1} K_{2}}\right) \text { is } 0(p) \text { as } p \rightarrow \infty .
\end{gathered}
$$

Proof: From Theorem 9 and (4.2.1)

$$
c(\beta, \delta) \leq k \exp \left(-\theta(\beta-1) /\left(K_{1} K_{2} \delta\right)\right)
$$

where $c(\beta, \delta)=\sup _{T} \sup _{\lambda>0} \frac{\mathbb{P}\left(X_{T} \geq \beta \lambda ; Y_{T} \leq \delta \lambda\right)}{\mathbb{P}\left(X_{T} \geq \lambda\right)}$.

If we now apply Theorem 10 with $\hat{\delta}=\frac{\theta}{K_{1} K_{2}}(\log k+(p+1) \log \beta$ ) (for suitably large $\beta$ ) and $F(x)=x^{p}$ we obtain

$$
\mathbb{E} X_{T}^{p} \leq\left(\frac{\beta}{\beta-1}\right)^{p+1}\left(\frac{\log k+(p+1) \log \beta}{\left(\theta / K_{1} K_{2}\right)}\right)^{p} \mathbb{E} Y_{T}^{p}
$$


and thus $\lim _{p \rightarrow \infty} \frac{C_{p}}{p} \leq\left(\frac{K_{1} K_{2}}{\theta}\right) \frac{\beta \log \beta}{\beta-1} \forall \beta>1$

4.3 Examples of pairs of functionals of Brownian motion which satisfy the conditions of Theorem 11 include

$\left.\begin{array}{llll}\text { (i) } & \left(B_{t}^{* 2}, t\right): & \theta=1 / 2 ; & K_{1}=1, K_{2}=1 \\ \text { (ii) } & \left(t, B_{t}^{* 2}\right): & \theta=\Pi^{2} / 8 ; & K_{1}=1, K_{2}=1 \\ \text { (iii) } & \left(l_{t}^{*}, B_{t}^{*}\right): & \text { for some } \theta ; & K_{1}=K_{2}=1 \\ \text { (iv) } & \left(B_{t}^{*}, l_{t}^{*}\right): & \text { for some } \theta ; & K_{1}=K_{2}=1\end{array}\right\} \begin{aligned} & \text { (see Jacka and Roberts } \\ & \text { (1988) for details) } \\ & \text { (see Barlow, Jacka and } \\ & \text { Yor (1986) for details) }\end{aligned}$

Note that (i) implies that

$$
\left\|B_{T}^{*}\right\|_{p} \leq C_{p}\left\|T^{1 / 2}\right\|_{p} \forall \text { optional } T
$$

with $C_{p}=0\left(p^{1 / 2}\right)$, a result not proved in any of the papers establishing (4.3.1) except Davis (1976).

Note that, following the method used in Bass (1987) we can establish similar results connecting $\left\|R_{\alpha, T}\right\|_{p}$ and $\left\|Y_{T}\right\|_{p}$ where $R_{\alpha, t} \equiv X_{t}^{\alpha+1} / Y_{t}^{\alpha}(\alpha \geq 0)$, and $X$ and $Y$ satisfy the conditions of Theorem 11.

\section{References}

[1] J.Azéma,R.F.Gundy and M.Yor: Sur l'intégrabilité uniforme des martingales continues. Sém. de Prob. XIV, LNM 784, Springer (1980).

[2] R. Bass: $L_{p}$-inequalities for functionals of Brownian motion. Sém. de Prob. XXI, LNM 1247, Springer (1987).

[3] M.T. Barlow, S.D. Jacka and M. Yor: Inequalities for a pair of processes stopped at a random time. Proc. LMS, (3), 52 (1986),142-172.

[4] M.T. Barlow and M. Yor: Semi-martingale inequalities via the Garsia-RodemichRumsey Lemma and applications to local times. J. Func. Anal, 49 (1982), 198-229.

[5] D.L.Burkholder: Distribution function inequalities for martingales. Ann. Prob. ,1 (1973),19-42.

[6] B. Davis: On the $L_{p}$-norms of stochastic integrals and other martingales. Duke Math. J., 43 (1976), 697-704.

[7] B. Davis: On the Barlow-Yor inequalities for local time. Sém. de Prob. XXI, LNM 1247, Springer (1987).

[8] S.D. Jacka and G.O. Roberts: Conditional diffusions: their generators and limit laws. Warwick research report no. 127 (1988).

[9] E. Lenglart, D. Lépingle and M. Pratelli: Présentation unifiée de certaines inégalités de la théorie des martingales. Sém. de Prob. XIV, LNM 784, Springer (1980). 